\title{
POINTS OF CONTINUITY FOR SEMIGROUP ACTIONS
}

BY

\author{
JIMMIE D. LAWSON ${ }^{1}$
}

\begin{abstract}
The purpose of this paper is to provide a more unified approach to questions involving the existence of points of joint continuity in separately continuous semigroup actions by deriving a small number of general principles which suffice to deduce previously derived results and generalizations thereof. The first major result gives sufficient conditions for a point to be a point of joint continuity in a general setting of "migrants", a useful symmetric generalization of semigroup actions. Results concerning actions of semigroups with group-like properties follow. In the latter part of the paper the notion of a subordinate point is introduced and joint continuity at subordinate points for various settings is proved. Finally, these results are applied to linear actions on locally convex spaces.
\end{abstract}

1. Introduction. In $1957 \mathrm{R}$. Ellis [6] showed that if the multiplication of a group on a locally compact space was separately continuous, then it was jointly continuous. By extending the techniques of R. Ellis, the author was able to show continuity at 1 (more generally, at units) in the semigroup setting [12]. At approximately the same time I. Namioka $[\mathbf{1 6}]$ proved a fundamental theorem concerning the existence of points of continuity for separately continuous functions with the second factor compact. This allowed the results on semigroup actions to be extended and the proofs to be simplified [13].

Recently there has been a renewed burst of activity in this area. D. Helmer $[\mathbf{8}, \mathbf{9}]$ extended the results of $[\mathbf{1 3}]$ and applied his results to actions of semigroups on linear spaces. J. P. Troallic $[\mathbf{2 6 , 2 7}$ introduced some novel ideas to deduce a variety of results -in particular, that the minimal ideal of a group-dense compact semitopological semigroup is a group. G. L. G. Sleijpen introduced a new class of semigroups called "stips" [23], and he and H. Dzinotyiweyi [5] proved several results concerning actions on the measure algebra in particular and on linear spaces in general.

In another direction W. Ruppert [17] studied continuity in semigroups with one-sided continuity. He has also exploited continuity at the units to introduce the machinery of group actions in order to obtain quite striking results on the structure of compact semitopological semigroups on manifolds $[\mathbf{1 9}]$ and those that have a dense subgroup [18]. More recently J. Berglund [1] has utilized the presence of continuity at the units in his study of weak periodicity. Thus not only have

Received by the editors September 7, 1983. Parts of this paper were presened on January 27, 1984, at the 90th Annual Meeting of the American Mathematical Society in Louisville, Kentucky. 1980 Mathematics Subject Classification. Primary 54H15.

Key words and phrases. Transformation semigroup, separable continuity, point of joint continuity, linear action.

${ }^{1}$ The author gratefully acknowledges the support of NSF Grant MCS-7900 295. 
the results on continuity per se expanded, but they are also playing an increasingly prominent role in the topics of semitopological semigroups, weak periodicity, analysis on semigroups, etc.

The purpose of this paper is to provide a more unified approach to continuity problems by deriving a small number of general principles which suffice to deduce many of the previously mentioned results and generalizations thereof. In $\S 3$ the Troallic transport principle is presented in the general setting of "migrants"; migrants are introduced because they give a useful "symmetric" generalization of semigroup actions. A sampling of a variety of consequences appears in $\S \S 4$ and 5 .

In $\S 6$ semigroups satisfying versions of Sleijpen's "exchange" axioms are introduced. The second major group of results, which concern actions of such semigroups, are given in $\S 7$. The arguments involve appropriately modified versions of the transport argument from my earlier paper [13]. It follows that such semigroups act continuously on compact spaces whenever the action is separately continuous; important generalizations of this result are also given.

The machinery of $\S 7$ is carried over to migrants in $\S 8$. Helmer's skillful techniques for using the theory of semigroup actions to study linear actions on locally convex spaces [9] are then employed in $\S 9$ to derive generalizations of results of Helmer, Sleijpen $[\mathbf{2 3}, \mathbf{2 5}]$, and Dzinotyiweyi [5] concerning linear actions of semigroups.

Throughout the paper the work of Namioka [16] forms the topological foundation for the results derived herein.

The author gratefully acknowledges the support of the Alexander von Humboldt Stiftung during the 1980-81 academic year at the Technische Hochschule Darmstadt. Some of the preliminary versions of results in the first part of this paper were obtained at that time. The author's awareness of and interest in questions concerning actions of semigroups on linear spaces were sparked by a visit of Heneri Dzinotyiweyi in the fall of 1982.

2. Preliminaries. Let $E$ be a set and let $\mathcal{V}, \mathcal{W} \subset E \times E$. We adopt the following standard notation:

$$
\begin{aligned}
\mathcal{V}^{-1} & =\{(x, y):(y, x) \in \mathcal{V}\}, \\
\mathcal{V} \circ \mathcal{W} & =\{(x, z):(x, y) \in \mathcal{V},(y, z) \in \mathcal{W} \text { for some } y \in E\}, \\
\mathcal{V}[x] & =\{y:(x, y) \in \mathcal{V}\} .
\end{aligned}
$$

Let $Z$ be a set and let $\Omega$ be a uniformity on $Z$. For another set $Y$, let $Z^{Y}$ denote the set of all functions from $Y$ to $Z$. For $A \subset Y$ and $W \in \Omega$ we define

$$
A \rightarrow \mathcal{W}=\left\{(f, g) \in Z^{Y} \times Z^{Y}:(f(x), g(x)) \in \mathcal{W} \text { for all } x \in A\right\} .
$$

The sets $A \rightarrow \mathcal{W}, \mathcal{W} \in \Omega$, form a basis for a uniformity of $Z^{Y}$, called the uniformity of uniform convergence on $A$.

Throughout the remainder of the paper all spaces are assumed to be Hausdorff. Let $X$ and $Y$ be topological spaces, let $(Z, \Omega)$ be a separated uniform space with uniformity $\Omega$, and let $*: X \times Y \rightarrow Z$ be a function sending $(x, y) \rightarrow x * y$. By means of the function $*$, we can alternately regard each member of $X$ as a function from $Y$ into $Z$. Specifically for $x \in X$, define $\hat{x}: Y \rightarrow Z$ by $\hat{x}(y)=x * y$. The function $x \rightarrow \hat{x}$ then defines a function from $X$ to $Y^{Z}$. 
2.1 Definition. For $\mathcal{W} \in \Omega$ and $A \subset Y$, we say that $x \in X$ is uniformly $\mathcal{W}$ continuous on $A$ if there exists an open neighborhood $N$ of $x$ such that $(x * y, u *$ $y) \in \mathcal{W}$ for all $u \in N, y \in A$. Equivalently $x$ is in the interior of $(A \rightarrow \mathcal{W})[x]$ where

$$
(A \rightarrow \mathcal{W})[x]=\{u \in X:(x * y, u * y) \in \mathcal{W} \text { for all } y \in A\} .
$$

We say $x$ is uniformly continuous on $A$ if $x$ is uniformly $\mathcal{W}$-continuous on $A$ for all $W \in \Omega$.

We say that $x \in X$ is jointly $\mathcal{W}$-continuous on $A$ if $x_{\alpha} \rightarrow x$ and $y_{\alpha} \rightarrow y$ in $A$, then for large $\alpha,\left(x_{\alpha} * y_{\alpha}, x * y\right) \in \mathcal{W}$.

The next proposition is standard and straightforward.

2.2 Proposition. Suppose that $*: X \times Y \rightarrow Z$ is separately continuous, that $A \subset Y$ is compact, and that $\mathcal{U}, \mathcal{W} \in \Omega$ satisfy $\mathcal{U} \circ \mathcal{U} \subset \mathcal{W}$.

If $x \in X$ is uniformly (jointly) $U$-continuous on $A$, then it is jointly (uniformly) $\mathcal{W}$-continuous on $A$.

We recall a (slightly modified) definition from [20] (see also the notion of JCC in $[\mathbf{9}])$.

2.3 Definition. A space $X$ is called a Namioka space if given any compact space $Y$, any uniform space $(Z, \Omega)$, and any separately continuous mapping $*: X \times Y \rightarrow$ $Z$, then for each $\mathcal{W} \in \Omega$ the set of points in $X$ which are (uniformly or jointly) $\mathcal{W}$-continuous on $Y$ has dense interior. (In light of Proposition 2.2, it makes no difference which type of $\mathcal{W}$-continuity is used in the definition.)

I. Namioka [16] made a major contribution to this area by showing that strongly countably complete regular spaces, a generalization of both complete metric and locally compact spaces, are Namioka spaces. Alternate proofs and generalizations have been given by Saint Raymond [20], Troallic [26], Kenderov [11], Helmer [9], and Christensen [3]. These papers (and others by these authors) contain several interesting applications of this important result. For the record we specify one equivalent version of Namioka's result [16] (see also [26, Theorem 4]).

2.4 THEOREM. If $X$ is a strongly countable complete regular Hausdorff space (in particular if $X$ is locally compact Hausdorff or complete metric), then $X$ is a Namioka space.

3. Migrants and the first transport technique. In this section a standard techique for obtaining specific points of continuity is introduced, what Ruppert has labeled a "transport" technique. The rough idea is to take points of continuity that are already known to exist (usually an amorphous lump of points guaranteed by Baire-categorical considerations) and "transport" the continuity to the desired point or points. In this section we adapt a technique of Troallic [27, 7]; in latter sections other techniques will be introduced.

We assume throughout this section the following situation: Let $X, Y$, and $Z$ be Hausdorff topological spaces, and let $\Pi: X \times Y \rightarrow Z$ be a function which sends $(x, y)$ to $x * y$. Let $M$ be a set (or space) of "migrants", and let $F: X \times M \rightarrow X$ sending $(x, m)$ to $x m$ and $G: M \times Y \rightarrow Y$ sending $(m, y)$ to $m y$ be functions such that for all $x \in X, m \in M$ and $y \in Y$ the following "transposition" law is satisfied:

$$
x *(m y)=(x m) * y .
$$


We normally assume that juxtapositions are performed first and omit the parentheses.

3.1 Definition. For $K \subset Y$, let $\Pi_{K}: X \times K \rightarrow Z$ denote the restriction of $\Pi$ to $X \times K$. A pont $x \in X$ is called a point of continuity of $\Pi_{K}$ if $\Pi_{K}$ is continuous at $(x, y)$ for all $y \in K$.

The following results were inspired by the work of Troallic [27], particularly his clever method of shifting between variables in separate-joint continuity arguments.

3.2 Lemma. Let $(Z, \Omega)$ be a uniform space, and assume $p \in X, K \subset Y$ satisfy the following hypotheses:

$\mathrm{H}_{1}$ : For all $m \in M$, the function $F_{m}: X \rightarrow X$ sending $x$ to $x m$ is continuous at p.

$\mathrm{H}_{2}$ : There exists $L \subset Y$ with the property that given $m \in M, y \in K$ there exists $z \in L$ such that $x * y=x * m z$ for all $x \in X$.

$\mathrm{H}_{3}$ : Given $\mathcal{U} \in \Omega$, there exists $x$ in the closure of $p M$ such that $x$ is uniformly $U$-continuous on $L$.

Conclusion: Then $p$ is uniformly continuous on $K$.

Proof. Let $\mathcal{W} \in \Omega$. Pick a symmetric $\mathcal{U} \in \Omega$ such that $U \circ U \subset \mathcal{W}$. By hypothesis there exists $x \in(p M)^{-}$such that $x$ is uniformly $\mathcal{U}$-continuous on $L$. Hence there exists an open neighborhood $U$ of $x$ such that for all $u \in U$ and $y \in L$, we have $(x * y, u * y) \in \mathcal{U}$. Choose $m \in M$ such that $p m \in U$. By hypothesis there exists an open neighborhood $N$ of $p$ such that $N m \subset U$.

To complete the proof, we show by means of the anticipated transposition argument that $(p * y, r * y) \in \mathcal{W}$ for all $r \in N, y \in K$. Since $y \in K$, there exists $z \in L$ such that $w * y=w * m z$ for all $w \in X$. Then $p * y=p * m z=p m * z$ and similarly for $r \in N, r * y=r m * z$. Since $N m \subset U,(x * z, p m * z) \in \mathcal{U}$ and $(x * z, r m * z) \in \mathcal{U}$. Thus $(p * y, r * y) \in \mathcal{U} \circ \mathcal{U} \subset \mathcal{W}$.

The following theorem is one important application of Lemma 3.2.

3.3 TheOREM. Let $X$ be a Namioka space, $Y$ a compact space, and $Z$ a uniform space. Assume further that II: $X \times Y \rightarrow Z$ is separately continuous and $x \rightarrow$ $x m: X \rightarrow X$ is continuous for all $x \in X$. Suppose also that $M$ satisfies either ( $\alpha) m Y=Y$ for all $m \in M$, or $(\beta)$ given an $m \in M$, there exists $m^{\prime} \in M$ such that $(x m) m^{\prime}=x$ for all $x \in X$. Then if $p \in X$ has the property that int $\left((p M)^{-}\right) \neq \emptyset$ (e.g. $p M$ is dense), then $p$ is a point of continuity for $\Pi$.

ProOF. Let $K=L=Y$ in Lemma 3.2. The first hypothesis of the lemma is clearly satisfied.

Let $m \in M$ and $y \in Y$. If $(\alpha)$ holds, then $y=m z$ for some $z \in Y$, so $x * y=$ $x * m z$ for all $x$. If $(\beta)$ holds, then let $z=m^{\prime} y$. We have $x * y=(x m) m^{\prime} * y=$ $x m * m^{\prime} y=x m * z=x * m z$ for all $x \in X$. Thus $\mathrm{H}_{2}$ holds.

Let $\mathcal{U} \in \Omega$. Since $X$ is a Namioka space, there exists a dense open set $V \subset X$ such that $x$ is uniformly $\mathcal{U}$-continuous on $Y$ for all $x \in V$. Then $W=\operatorname{int}\left((p M)^{-}\right) \cap V$ is nonempty and open. Hence the third hypothesis is satisfied. By Lemma $3.2 p$ is uniformly continuous on $Y$. It follows from Proposition 2.2 that $p$ is a point of continuity for $\Pi$.

4. Group closures. In this section we apply the results of the previous section to compact semigroups with a separately continuous multiplication and a dense 
subgroup. A prime example is the weakly almost periodic compactification of a topological group.

Throughout this section let $S$ be a compact Hausdorff semigroup with separately continuous multiplication (i.e. a compact semitopological semigroup) and let $H$ be a subgroup such that $\bar{H}=S$. Note that by continuity the identity 1 of $H$ is an identity for all of $S$. We employ Theorem 3.3 to study the structure of $S$.

4.1 Proposition. Let $x, s \in S$ and suppose $x s S=x S$. If $\left\{g_{\alpha} \in H: \alpha \in D\right\}$ is a net converging to $s$, then for any cluster point $t$ of $\left\{g_{\alpha}^{-1}\right\}, x s t=x$.

ProOF. Let $X=x S, M=H, Y=S, Z=S$ and the various functions of Theorem 3.3 be the appropriate restrictions of multiplication. Since $H$ is dense in $S, x s H$ is dense in $x s S=x S=X$. If $g \in H$, then $S=g g^{-1} S \subset g S$. Thus by Theorem 3.3 the restriction of multiplication $m: x S \times S \rightarrow S$ is continuous at $(x s, t)$. Now $x g_{\alpha} \rightarrow x s$ in $x S$ and $g_{\alpha}^{-1}$ clusters to $t$ in $S$. Hence $x=x g_{\alpha} g_{\alpha}^{-1}$ clusters to $(x s) t$. Thus $x=x s t$.

4.2 Notation. If $x \in S$, then $x^{\prime}$ will denote some (arbitrarily chosen) cluster point of $\left\{g_{\alpha}^{-1}\right\}$ where $\left\{g_{\alpha}\right\}$ is a net in $H$ converging to $x$.

We turn now to the study of regular elements in $S$. Recall from semigroup terminology. that an element $x \in S$ is regular if there exists $y \in S$ such that $x y x=x$ (see [4] for basic semigroup concepts).

4.3 Proposition. Let $x$ be a regular element of $S$. Then there exist unique idempotents $e, f$ such that e, resp. $f$, is $R$-equivalent, resp. L-equivalent, to $x$ (i.e. $e S=x S, S f=S x)$. Furthermore, $x x^{\prime}=e, x^{\prime} x=f$, and $x x^{\prime} x=x$.

Proof. Suppose $e=e^{2}$ and $e S=x S$. Since $e$ is a left identity for $e S, e x=x$. Thus $e S=x S=e x S$. By Proposition $4.1 e x x^{\prime}=e$. Hence $x x^{\prime}=e x x^{\prime}=e$. Dually $x^{\prime} x=f$. The existence of $e$ and $f$ follows from the regularity of $x$ (see e.g. $[4$, p. 58]), and the argument just given establishes their uniqueness. Finally $x x^{\prime} x=e x=x$.

4.4 REMARK. (i) By Proposition 4.3 each row and column of a regular D-class $D$ have only one idempotent. These idempotents may be used to index the rows and columns of $D$, and then the $H$-classes with idempotents are precisely those on the "diagonal".

(ii) If $x^{\prime}$ is also a regular element, then by the dual of Proposition $4.3, x^{\prime} x x^{\prime}=x^{\prime}$. Thus $x$ and $x^{\prime}$ are inverses. By standard semigroup theory $x$ has a unique inverse (since each $R$-class and $\mathcal{L}$-class in the $D$-class of $x$ have only one idempotent). Thus if $g_{\alpha} \rightarrow x$, then the net $\left\{g_{\alpha}^{-1}\right\}$ has at most one regular cluster point, namely the inverse of $x$.

(iii) If $S$ is the weakly almost periodic compactification of $H$, then by the universal property of $S$, the anti-involution $g \rightarrow g^{-1}$ on $H$ induces an anti-involution $I: S \rightarrow S$. By continuity if $g_{\alpha} \rightarrow x$, then $g_{\alpha}^{-1} \rightarrow I(x)$; furthermore, if $x$ is regular, then also $I(x)$ is (since $I$ is an anti-isomorphism). Thus by part (ii) of this remark $x$ and $I(x)$ are (the unique) inverses of each other. In particular, for an idempotent $e, I(e)=e$.

It appears to be an interesting line of investigation to study in greater detail properties of the involution $I$. 
4.5 COROLLARY. Let $T$ be a closed subsemigroup of a compact semigroup $S$ with a dense subgroup. Then the minimal ideal of $T$ is a group. In particular, the minimal ideal of $S$ is a group.

ProOF. It is well known and basic that the minimal ideal is completely simple (see e.g. [2, II.1]). By Proposition 4.3 each row and column of the minimal ideal has at most one idempotent. Hence the minimal ideal is a group.

The last part of Corollary 4.5 was an important early result in the theory of separately continuous semigroups; the early proof was an analytic one which employed the Ryll-Nardzewski fixed point theorem [20]. Troallic [27] has recently given a topological proof of this result in the same spirit as that given here.

The next result was first obtained by W. Ruppert (but after I had told him about the earlier results of this section).

4.6 COROLLARY. Suppose that,$f$ are idempotents in a group-dense semigroup $S$. If $e f=f$, then $f e=f$. Furthermore the idempotents $E(S)$ of $S$ form a complete lattice with respect to the usual natural partial ordering of idempotents.

Proof. Suppose $e f=f$ for $e, f \in E(S)$. A routine verification yields that $f e$ is an idempotent and $f S=f e S$. By Proposition $4.3 f=f e$.

Let $A$ be a nonempty subset of idempotents and let $T$ be the smallest closed subsemigroup containing $A$. By Corollary 4.5 the minimal ideal of $T$ is a group; let $f$ be the idempotent in this group. We establish that $f$ is the greatest lower bound for $A$ in $E(S)$.

Let $e \in A$. Then $e f$ is in the minimal ideal of $T$; hence $f(e f)=e f$. Thus $(e f)^{2}=e(f(e f))=e e f=e f$, i.e. $e f$ is an idempotent. Thus $e f=f$ (since $f$ is the unique idempotent in the minimal ideal). By the first part of the proof $f=f e$. Thus $f \leq e$. It follows that $f$ is a lower bound for $A$.

Let $g$ be another lower bound. Then $g e=g$ for all $e \in A$. Thus the closed subsemigroup $U=\{x \in S: g x=g\}$ contains $A$ and hence $T$. We conclude $g f=g$. Similarly $f g=g$. It follows that $g \leq f$, i.e. $f$ is the greatest lower bound for $A$.

Since $E(S)$ has a largest element 1 and every nonempty set has a greatest lower bound, it follows that $E(S)$ is a complete lattice.

5. Group and semigroup actions. We turn now to the application of $\S 3$ to group and semigroup actions. We begin with a mild generalization of a theorem of Ruppert [19, Theorem II-4.11]. If $S$ is a semigroup, an element $s \in S$ is called a right unit if $s S=S$. Note that a unit in a semigroup with 1 is a right unit.

5.1 THEOREM. Let $S$ be a semigroup on a compact space such that the set of right units is dense, let $X$ be a compact space, and let $\Pi: X \times S \rightarrow X$ be a separately continuous right action of $S$ on $X$.

(i) If $\operatorname{int}(p S) \neq \emptyset($ e.g. $p S=X)$ for some $p \in X$, then $p$ is a point of continuity for $\Pi$.

(ii) If the action is minimal in the sense that $x S=X$ for all $x \in X$, then $\Pi$ is continuous.

Proof. (i) Apply Theorem 3.3 to $\Pi: X \times S \rightarrow S$ with $M$ the set of right units and the functions $X \times M \rightarrow X$ and $M \times S \rightarrow S$ the restrictions of the action and multiplication respectively. Now $\bar{M}=S$ implies $p S=p M^{-} \subset(p M)^{-}$. Thus $\operatorname{int}(p M)^{-} \neq \emptyset$. 
(ii) is immediate from (i).

By switching the roles of $X$ and $S$, we obtain one of the principal results of Hansel and Troallic [7]. The reader is referred there for some nice applications of this result.

5.2 THEOREM. Let $S$ be a semigroup on a Namioka space and suppose right translations by elements of $S$ are continuous. Let $\Pi: S \times Y \rightarrow Y$ be an action of $S$ on a compact space $Y$ and let $M$ denote the set of $s \in S$ such that $s Y=Y$. If $t \in S$ and the interior of $(t M)^{-}$is nonempty, then $t$ is a point of continuity for $\Pi$. In particular if $M$ is dense in $S$ and left translation by $t$ is continuous, then $t S=S$ implies $t$ is a point of continuity for $\Pi$.

Proof. Apply Theorem 3.3 with $X=S, M=M, Y=Y, Z=Y$, and $p=t$. To conclude that $M$ dense in $S$ implies $t M$ is dense in $t S=S$, we need that left translation by $t$ is continuous.

Namioka [16] and Troallic [26, Theorem 10] have given generalizations of the well-known theorem of Ellis on group actions [6]. The following result is in the same vein.

5.3 COROLlaRY. Let $S$ be a semigroup on a Namioka space such that right translations in $S(x \rightarrow x s)$ are continuous. Let $\Pi: S \times Y \rightarrow Y$ be a separately continuous action of $S$ on a locally compact space $Y$ such that $y \rightarrow s * y: Y \rightarrow Y$ is a homeomorphism for each $s \in S$. If $\operatorname{int}(t S)^{-} \neq \emptyset$, then $t$ is a point of continuity for $\Pi$.

ProOF. Let $X=M=S, Y^{\infty}=Y \cup\{\infty\}$ be the one point compactification of $Y$. The action $\Pi$ extends to a separately contionuous action of $S$ on $Y^{\infty}$ defined by $s * \infty=\infty$ (since each $s$ acts as a homeomorphism). The conclusion now follows from Theorem 5.2 .

5.4 COROLLARY. If $G$ is a group on a Namioka space in which right translations are continuous, and if $\Pi: G \times Y \rightarrow Y$ is a separately continuous action on a locally compact space $Y$, then $\Pi$ is continuous.

ProOF. Let $e$ be the identity of $G$. Then $e Y$ is a retract of $Y$, hence locally compact. By Corollary 5.3 the restriction $\Pi: G \times e Y \rightarrow e Y$ is continuous. Hence the composition $(g, y) \rightarrow(g, e * y) \rightarrow g *(e * y)=g * y=\Pi(g, y)$ is continuous.

6. Subordinate points. We introduce in this section certain classes of semigroups and certain types of points in semigroups which exhibit desirable behavior in our study of actions in later sections. The definitions themselves are variations on a theme of Sleijpen $[\mathbf{2 3}, \mathbf{2 5}]$.

6.1 Definition. (i) Let $S$ be a semitopological monoid. A point $s \in S$ is called a point of weak subordination (or a weakly subordinate point) if given any neighborhood $U$ of 1 then

$$
U_{s}=\{t \in S: U s U \cap U t \neq \emptyset\}
$$

is a neighborhood of $s$.

The monoid $S$ is called a semigroup with identity dominant (abbreviated "swid") if every point of $S$ is a weakly subordinate point and if multiplication is continuous at $(1, x)$ for all $x \in S$. 
(ii) Let $S$ be a semitopological semigroup. A point $s \in S$ is called a point of subordination (or subordinate point) if given an open set $U \neq \emptyset$,

$$
U^{-1}(U s)=\{t \in S: U t \cap U s \neq \emptyset\}
$$

is a neighborhood of $s$.

The semigroup $S$ is called a semigroup with all subordinate points (abbreviated "swasp") if every point is subordinate.

(iii) Let $S$ be a semitopological semigroup. A point $s \in S$ is called a point of strong subordination (or a strongly subordinate point) if given any open set $U \neq \emptyset$, there exists $u \in U$ such that

$$
u^{-1}(U s)=\{t \in S: u t \in U s\}
$$

is a neighborhood of $s$.

6.2 LEMMA. Each corresponding pair of the following are equivalent:

(1) The point $s$ is a weakly subordinate (resp. subordinate) [resp. strongly subordinate] point.

(2) If $s_{\alpha} \rightarrow s$, then there exists a subnet $s_{\beta}$ and nets $u_{\beta}, v_{\beta}, w_{\beta} \rightarrow 1$ such that $u_{\beta} s_{\beta}=v_{\beta} s w_{\beta}$ for all $\beta$ (resp. if $s_{\alpha} \rightarrow s$ and $t \in S$, then there exists a subnet $s_{\beta}$ and $u_{\beta}, v_{\beta} \rightarrow t$ such that $u_{\beta} s_{\beta}=v_{\beta}$ sor all $\beta$ ) [resp. if $s_{\alpha} \rightarrow s$ and $U \neq \emptyset$ is open, then there exists $u \in U$ and $\left\{v_{\alpha}\right\} \subset U$ such that $u s_{\alpha}=v_{\alpha} s$ for large $\alpha$ ). (Note that in the third case the net $\left\{v_{\alpha}\right\}$ need not converge.)

Proof. (1) implies (2): Let $s_{\alpha} \rightarrow s$. Form the directed set $\beta=(\alpha, U)$ where $U$ ranges over the neighborhoods of 1 directed by reverse inclusion and $s_{\alpha} \in U_{s}$. If $\beta=(\alpha, U)$, let $s_{\beta}=s_{\alpha}$, and choose $u_{\beta}, v_{\beta}, w_{\beta} \in U$ such that $u_{\beta} s_{\beta}=v_{\beta} s w_{\beta}$.

(2) implies (1): Suppose there exists a neighborhood $U$ of 1 such that $U_{s}$ is not a neighborhood of $s$. Then there exists $s_{\alpha} \rightarrow s$ such that $s_{\alpha} \notin U_{s}$ for all $\alpha$. Pick a subset $s_{\beta} \rightarrow s$ and $u_{\beta}, v_{\beta}, w_{\beta} \rightarrow 1$ such that $u_{\beta} s_{\beta}=v_{\beta} s w_{\beta}$ for all $\beta$. Eventually $u_{\beta}, v_{\beta}, w_{\beta} \in U$, so $s_{\beta} \in U_{s}$, a contradiction.

The two alternate equivalences involve similar arguments and hence are omitted.

We shall employ freely the alternate characterizations of Lemma 6.2 for various points of subordination. We henceforth denote subnets with the same subscripts.

6.3 Proposition. (i) Let $S$ be a semitopological monoid. A point $s \in S$ is a point of subordination if and only if $U^{-1}(U s)$ is a neighborhood of $s$ for every neighborhood $U$ of 1 . Hence a subordinate point is weakly subordinate.

(ii) In a semitopological semigroup a strongly subordinate point is subordinate.

PROOF. (i) Suppose $U^{-1}(U s)$ is a neighborhood of $s$ whenever $U$ is a neighborhood of 1 . Let $V$ be a nonempty open set and pick $r \in V$. There exists an open set $U$ containing 1 such that $r U \subset V$. Then $U t \cap U s \neq \emptyset$ implies $r U t \cap U s \neq \emptyset$ implies $V t \cap V s \neq \emptyset$. Hence $V^{-1}(V s)$ is a neighborhood of $s$. The converse is trivial. For a neighborhood $U$ of $1, U t \cap U s \neq \emptyset$ since $U s=U s 1 \subset U s U$. Thus a subordinate point is weakly subordinate.

Part (ii) is immediate.

In [3] the notion of a "substantial" point was introduced (see [9] for variants). A point $s \in S$ is substantial if given a closed proper (possibly empty) left ideal $L$ of $S$ and an open cover $B$ of $S \backslash L$, there exist $B \in B$ such that $\operatorname{int}(B s) \neq \emptyset$. (This is actually slightly less general than the definition in [13 and 9], but agrees in the locally compact case.) 


\subsection{PRoposition. A substantial point is a point of strong subordination.}

Proof. Let $s \in S$ be a substantial point. Set

$$
\begin{aligned}
& L(s)=\{t \in S: \text { for every neighborhood } U \text { of } t \text {, there exists } \\
& \left.\qquad u \in U \text { such that } u^{-1}(U s) \text { is a neighborhood of } s\right\} .
\end{aligned}
$$

It follows using separate continuity that $L(s)$ is a closed left ideal of $S$. If $L(s) \neq S$, then for each $x \notin L(s)$, choose a neighborhood $U(=U(x))$ of $x$ such that for all $u \in U, u^{-1}(U s)$ is not a neighborhood of $s$. By hypothesis there exists some $U=U(x)$ such that $\operatorname{int}(U s) \neq \emptyset$.

Let $u \in U$ such that $u s \in \operatorname{int}(U s)$. If $s_{\alpha} \rightarrow s$, then $u s_{\alpha} \rightarrow u s$, so eventually $u s_{\alpha} \in U s$, a contradiction. Hence $L(s)=S$ and $s$ is a point of strong subordination.

The reader is referred to $[\mathbf{1 3}]$ for special classes of substantial points (and hence, in the locally compact case, of subordinate points). In general subordinate points occur more frequently than substantial points.

It appears worthwhile to put on record a little concerning swasps [swids]. They are generalizations of Sleijpen's notion of a stip, and include all discrete semigroups [monoids], subsemigroups [submonoids] $S$ of topological groups for which the interior of $S$ is dense in $S$ (e.g. finite-dimensional cones), and semigroups [monoids] in which right translations are open mappings (see $[\mathbf{2 3}, \mathbf{2 4}]$ for more examples of stips, and hence swasps and swids).

The following closure properties allow the construction of further examples. (Indeed it was the desire for closure with respect to products that motivated the introduction of swids.)

6.5 Proposition. Let $S$ be a product of semitopological monoids. A point $s \in S$ is a point of weak subordination if and only if each coordinate is. Hence a product of swids is a swid.

ProOF. Let $S=A \times B \times C \times \cdots$ be a product of monoids for some (possibly infinite) alphabet (without $S$ ). Let $s=(a, b, \ldots) \in S$ with each coordinate a point of weak subordination. Let $U \times B \times C \times \cdots$ be a subbasic open set containing 1 . Then $N=U_{a} \times B \times C \times \cdots$ is a neighborhood of $s$, and if $\left(a_{1}, b_{1}, c_{1}, \ldots\right) \in N$, then $u a_{1}=v a w$ for some $u, v, w \in U$ and $b b_{1}=1(b) b_{1}$ etc. in the other coordinates. This argument holds also for basic open sets.

The converse statement is straightforward. Note that the appropriate continuity requirements at 1 are preserved by products. Hence a product of swids is a swid.

By taking infinite products of certain nonabelian finite monoids, one obtains examples of swids that are not swasps. Infinite products of abelian swasps are again swasps; these products contain many subordinate points that are not substantial points.

Recall that a surjective mapping $f: X \rightarrow Y$ is called pseudo-open if given $y \in Y$ and $U$ open containing $f^{-1}(y)$, then $f(U)$ is a neighborhood of $y$. Both closed mappings and open mappings are pseudo-open, and these in turn are quotient mappings.

6.6 PROPOSITION. If $f: S \rightarrow T$ is a continuous, pseudo-open homomorphism from $S$ onto $T$, and if $s \in S$ is a subordinate point, then so is $f(s)$. Hence if $S$ is a swasp, so is $T$. 
Proof. Let $t \in T$ and let $V \neq \emptyset$ be open in $T$. Let $U=f^{-1}(V)$. Then

$$
N=\bigcup\left\{U^{-1}(U s): s \in f^{-1}(t)\right\}
$$

is a neighborhood of $f^{-1}(t)$. Hence by hypothesis $f(N)$ is a neighborhood of $t$. If $b=f(a)$ for $a \in N$, then $u a=v s$ for some $u, v \in U$ and $s \in f^{-1}(t)$. Thus $f(u) b=f(v) t$, i.e. $b \in V^{-1}(V t)$.

6.7 REMARK. The preceding proof goes through for weakly and strongly subordinate points. In order for $T$ to be a swid, one needs additionally continuity at 1 . This will follow if $S$ is a locally compact, $\sigma$-compact topological semigroup, since $T$ is also a topological semigroup in this case $[\mathbf{1 4}, \mathrm{p}$. 21]. With slight modification of the preceding proof one can obtain an analogue of the proposition for Sleijpen's stips.

Since the Rees quotient $S \rightarrow S / I$ obtained by collasping a closed ideal is a closed mapping, Rees quotients of swasps are swasps (e.g. $\mathbf{R}^{+} \rightarrow \mathbf{R}^{+} /[1, \infty)$; the quotient is sometimes called the "nil-interval" since every nonidentity element is nilpotent).

7. Transport-exchange arguments. In this section a second transport technique is introduced. The transport idea appears implicitly in the work of Ellis [6] and later becomes explicit in earlier work of the author $[\mathbf{1 2}, \mathbf{1 3}]$. Refinements and applications appear in the work of Ruppert [17] and Helmer [9]. The arguments found here involve situations where convergence satisfies a certain "exchange" property reminiscent of the work of Sleijpen.

A second transport, which occurs only in monoids, involves transporting continuity at the identity to subordinate points.

7.1 DEFinition. Let $\Pi: S \times Y \rightarrow Y$ be an action of a semitopological semigroup $S$ on a space $Y$. The action $\Pi$ is said to be locally reductive at $s$ if given $s_{\alpha} \rightarrow s$ in $S, s_{\alpha} y_{\alpha} \rightarrow z$ in $Y$, and $s y \neq z$, then $t s y \neq t z$ for some $t \in S$. The action $\Pi$ is locally reductive if it is locally reductive at all $s \in S$.

7.2 Proposition. If $S=\bigcup_{i=1}^{n} e_{i} S$, where $e_{i}$ is an idempotent for each $i$, then any separately continuous action $\Pi: S \times Y \rightarrow Y$ is locally reductive. In particular if $S$ has a left identity, such actions are locally reductive.

PROOF. If $r \in e_{i} S$, then $e_{i} r=r$. Hence for a cofinal subset of the $s_{\alpha}$, there exists an idempotent $e$ such that $e s_{\alpha}=s_{\alpha}$ for each $\alpha$. By separate continuity in $S$, $e s=s$. Thus $e s y \neq s y$. Since $s_{\alpha} y_{\alpha}=e s_{\alpha} y_{\alpha} \rightarrow e z$, we have $z=e z$. Thus $e s y=e z$.

There are other hypotheses that give rise to locally reductive actions, but this is a topic which we do not choose to pursue here.

Before considering actions in some detail, we have need of some additional concepts related to functions on product spaces. We begin with a lemma.

7.3 LEMMA. The following conditions on a function $H: X \times B \rightarrow Z$ are equivalent:

(1) Given $a_{\alpha} \rightarrow a$ in $X, b_{\alpha} \rightarrow b$ in $B$, and $H\left(a_{\alpha}, b_{\alpha}\right) \rightarrow z \in Z$, then $z=H(a, b)$.

(2) Given $z \neq H(a, b)$, there exist open sets $U, V, W$ with $a \in U, b \in V, z \in W$ and $H(U \times V) \cap W=\emptyset$.

(3) Given a compact set $K \subset Z \backslash\{H(a, b)\}$, there exist open sets $U, V, W$ with $a \in U, b \in V, K \subset W$, and $H(U \times V) \cap W=\emptyset$.

PROOF. Straightforward. 
We remark that equivalence (1) is satisfied for all $(a, b) \in X \times B$ if and only if $H$ has closed graph. Hence (1) gives a local version of closed graph.

7.4 Definition. Let $H: X \times Y \rightarrow Z$ be a function between spaces. Let $K \subset Y$, and let $H_{K}: X \times K \rightarrow Z$ denote the restriction of $H$. We say that $H_{K}$ is $C$ continuous at $(a, b) \in X \times K$ if any of the three equivalent conditions of Lemma 7.3 are satisfied $(C$-continuous stands for continuous with respect to the compact sets as in Lemma 7.3(3)). We say $x \in X$ is a point of $C$-continuity for $H_{K}$ if $H_{K}$ is $C$-continuous at $(x, y)$ for all $y \in K$.

Let $K$ be a collection of subsets of $Y$. We say that $H$ is locally bounded at $x$ with respect to $K$ if given $K \in \mathcal{K}$, there exists a neighborhood $U$ of $x$ such that $H(U \times K)$ has compact closure. If $K$ is the collection of compact sets, we say simply that $H$ is locally bounded at $x$.

The first part of the following theorem is a variant of earlier results of myself [13], Helmer [9, Theorem 2.5(2)] and Hansel and Troallic [7, Theorem 5.2].

7.5 THEOREM. Let $S$ be a semitopological semigroup, and suppose $\Pi: S \times Y \rightarrow$ $Y$ is a separately continuous action of $S$ on a separated uniform space $Y$.

(i) If $S$ is a Namioka space and has an identity 1 , and if $K \subset Y$ is compact, then 1 is a point of $C$-continuity for $\Pi_{K}$.

(ii) If $S$ is a Namioka space, if $s \in S$ is a subordinate point, if the action $\Pi$ is locally reductive at $s$, and if for a compact set $K \subset Y$, either $\Pi$ is locally bounded at $s$ with respect to $\{K\}$ or $Y$ is locally compact, then $s$ is a point of $C$-continuity for $\Pi_{K}$.

(iii) Let $S$ be a monoid, and suppose $s \in S$ is a weakly subordinate point. If for $A \subset Y$, there exist a neighborhood $U$ of 1 , a neighborhood $N$ of $s$, and a closed set $B \subset Y$ such that $U A \cup N A \subset B$ and if 1 is a point of continuity for $\Pi_{B}: S \times B \rightarrow Y$, then $s$ is a point of $C$-continuity for $\Pi_{A}$.

ProOF. (i) Let $u_{\alpha} \rightarrow 1$ in $S, y_{\alpha} \rightarrow y$ in $K$, and suppose $u_{\alpha} y_{\alpha} \rightarrow z \neq 1 y$. Then $u_{\alpha} y_{\alpha}=1 u_{\alpha} y_{\alpha} \rightarrow 1 z$ implies $z=1 z$. There exists $\mathcal{W} \in \Omega$, the uniformity on $Y$, such that $\mathcal{W}[1 y] \cap \mathcal{W}[z]=\emptyset$. Pick a symmetric $U \in \Omega$ such that $U \circ U \subset \mathcal{W}$. There exists an open set $N$ containing 1 such that $N y \subset \mathcal{U}[1 y]$ and $N z \subset \mathcal{U}[z]$. Since $S$ is a Namioka space, there exists $r \in N$ such that $r$ is jointly $U$-continuous on $K$. Since $r u_{\alpha} \rightarrow r$, we have eventually $\left(\left(r u_{\alpha}\right) y_{\alpha}, r y\right) \in \mathcal{U}$. Also $r \in N$ implies $(r y, 1 y) \in \mathcal{U}$. Thus eventually $\left(r u_{\alpha} y_{\alpha}, 1 y\right) \in \mathcal{U} \circ \mathcal{U} \subset \mathcal{W}$. On the other hand $u_{\alpha} y_{\alpha} \rightarrow z$ implies that eventually $\left(r\left(u_{\alpha} y_{\alpha}\right), r z\right) \in \mathcal{U}$, and $r \in N$ implies $(r z, z) \in \mathcal{U}$. Hence eventually $\left(r u_{\alpha} y_{\alpha}, z\right) \in \mathcal{W}$. But these statements contradict $\mathcal{W}[1 y] \cap \mathcal{W}[z]=\emptyset$.

(ii) Let $s_{\alpha} \rightarrow s$ in $S, y_{\alpha} \rightarrow y$ in $K$, and suppose $s_{\alpha} y_{\alpha} \rightarrow z \neq s y$. Since the action is locally reductive at $s$, there exists $h \in S$ such that $h z \neq h s y$. Pick symmetric entourages $\mathcal{U}, \mathcal{W} \in \Omega$ such that $\mathcal{U} \circ \mathcal{U} \subset \mathcal{W}$ and $\mathcal{W}[h z] \cap \mathcal{W}[h s y]=\emptyset$. There exists an open set $U$ containing $h$ such that $U z \subset \mathcal{U}[h z]$ and $U s y \subset \mathcal{U}[h s y]$. By local boundedness there exists a compact set $G$ and a neighborhood $N$ of $s$ such that $N K \subset G$. Since $S$ is a Namioka space, there exists $r \in U$ such that $r$ is $U$-continuous on $G$. By Lemma 6.2 there exist nets $u_{\alpha}, v_{\alpha} \rightarrow r$ such that $u_{\alpha} s_{\alpha}=v_{\alpha} s$. By $U_{-}$ continuity on $G$ we have eventually $\left(u_{\alpha}\left(x_{\alpha} y_{\alpha}\right), r z\right) \in \mathcal{U}$ and $\left(v_{\alpha}\left(s y_{\alpha}\right), r s y\right) \in \mathcal{U}$. Since $(r z, h z) \in \mathcal{U},(r s y, h s y) \in \mathcal{U}$, and $u_{\alpha} s_{\alpha} y_{\alpha}=v_{\alpha} s y_{\alpha}$, we conclude eventually $u_{\alpha} s_{\alpha} y_{\alpha} \in \mathcal{W}[h z] \cap \mathcal{W}[h s y]$, a contradiction.

The proof for the case $Y$ is locally compact is quite similar. In this case one picks the set $G$ to be the union of $s K$ and a compact neighborhood of $z$. 
(iii) Let $s_{\alpha} \rightarrow s, y_{\alpha} \rightarrow y$ in $A$, and $s_{\alpha} y_{\alpha} \rightarrow z$. By the definition of a subordinate point, there exists $u_{\alpha}, v_{\alpha}, w_{\alpha} \rightarrow 1$ such that $u_{\alpha} s_{\alpha}=v_{\alpha} s w_{\alpha}$ for all $\alpha$. Then $u_{\alpha}\left(s_{\alpha} y_{\alpha}\right) \rightarrow 1 z=z$ since eventually $s_{\alpha} y_{\alpha} \in N A \subset B, B$ closed implies $z \in B$, and $\Pi_{B}$ is continuous at $(1, z)$. But $u_{\alpha} s_{\alpha} y_{\alpha}=\left(v_{\alpha} s w_{\alpha}\right) y_{\alpha}=v_{\alpha}\left(s\left(w_{\alpha} y_{\alpha}\right)\right)$. Now eventually $w_{\alpha} y_{\alpha} \in U A \subset B$, so $w_{\alpha} y_{\alpha} \rightarrow 1 y$. By separate continuity $s\left(w_{\alpha} y_{\alpha}\right) \rightarrow s 1 y=s y$. Since $s w_{\alpha} \rightarrow s$, eventually $s w_{\alpha} y_{\alpha} \in N A \subset B$. Thus $z=s y$.

In light of Theorem 7.5 it is of interest to identify sufficient conditions for points of $C$-continuity to be points of continuity.

7.6 Proposition. Suppose $F: A \times B \rightarrow Z$ is separately continuous and is $C$ continuous at the point $(a, b)$. Then $F$ is continuous at $(a, b)$ if any of the following conditions are satisfied:

(i) There exist open sets $U, V$ such that $a \in U, b \in V$, and $F(U \times V)$ has compact closure;

(ii) $Z$ is compact;

(iii) $A$ is locally connected at a (or $B$ is locally connected at b) and $Z$ is locally compact (actually rim compact suffices).

ProOF. (i) Let $F(a, b) \in W$, an open set. Let $K=F(U \times V)^{-} \backslash W$; then $K$ is compact. By Lemma 7.3 there exist open sets $P, Q, a \in P \subset U, b \in Q \subset V$, with $F(P \times Q) \cap K=\emptyset$. Since $F(P \times Q) \subset F(U \times V)$, we conclude $F(P \times Q) \subset W$.

(ii) A special case of (i).

(iii) Let $W$ be a neighborhood of $F(a, b)$ with compact boundary $\operatorname{Bd}(W)$. Pick a connected neighborhod $N$ of $a$ and a neighborhood $V$ of $b$ so that $F(\{a\} \times V) \subset W$, $F(N \times V) \cap \operatorname{Bd}(W)=\emptyset$. Then if $v \in V, F(N \times\{v\})$ is connected and contains $(a, v) \in W$. Since $F(N \times\{v\})$ misses $\operatorname{Bd}(W)$, we conclude $F(N \times\{v\}) \subset W$. Since $v$ was arbitrary, $F(N \times V) \subset W$.

7.7 COROLlaRY. Let $S$ be a semitopological semigroup on a Namioka space, let $\Pi: S \times Y \rightarrow Y$ be a separately continuous action of $S$ on a separated uniform space $Y$, and let $\mathcal{K}$ be a collection of compact subsets of $Y$.

(i) If $s \in S$ is a subordinate point and if the action is locally reductive at $s$ and locally bounded at $s$ with respect to $K$, then $s$ is a point of continuity for $\Pi_{K}$ for all $K \in \mathcal{K}$.

(ii) If $S$ is a monoid for which multiplication is continuous at $\{1\} \times S$, if $s \in S$ is a weakly subordinate point, and if the action is locally bounded at 1 and $s$ with respect to $\mathcal{K}$, then $s$ is a point of continuity for $\Pi_{K}$ for all $K \in \mathcal{K}$.

PrOOF. (i) This part follows immediately from part (i) of Proposition 7.6 and part (ii) of Theorem 7.5.

(ii) Let $K \in \mathcal{K}$ and $y \in Y$. Pick $U$ open containing 1 and $Q$ open containing $s$ such that $U K \cup A K \subset G$ for $G$ compact. By continuity of multiplication at $(1,1)$ and $(1, s)$, there exists an open neighborhood $V$ of 1 and $N$ of $s$ such that $V V \subset U$ and $V N \subset Q$. Then $V(V K)=(V V) K \subset U K \subset G$ and similarly $V(N K) \subset G$. Let $B$ be the closure of $V K \cup N K$. Then $B$ is compact and by separate continuity $V B \subset G$.

By Theorem 7.5(i), 1 is a point of $C$-continuity for $\Pi_{B}$. Applying Proposition 7.6(i) to $\Pi_{B}: S \times B \rightarrow Y$, we conclude 1 is a point of continuity for $\Pi_{B}$ (since $V B \subset M)$. Since $V K \cup N K \subset B, s$ is a point of $C$-continuity for $\Pi_{B}$ (since 
$V B \cup M)$. Since $V K \cup N K \subset B, s$ is a point of $C$-continuity for $\Pi_{K}$ by Theorem 7.5(iii). Since $N K \subset B, s$ is a point of continuity for $\Pi_{K}$ by Proposition 7.6(i).

7.8 COROLlaRY. Let $S$ be a swid [swasp] on a Namioka space, $Y$ a uniform space, and $\Pi: S \times Y \rightarrow Y$ a separately continuous [locally reductive] action. Then $\Pi$ is continuous if any of the following conditions hold:

(i) $Y$ is locally compact and $S$ has a basis of connected neighborhoods at $1[S$ is locally connected];

(ii) $Y$ is locally compact and locally connected;

(iii) $\Pi$ is locally bounded at $s$ for all $s \in S$ and the topology of $S \times Y$ is the finest coherent with all rectangles $S \times K$ where $K \subset Y$ is compact. The latter will hold in particular $(\alpha)$ if $S$ is locally compact and $Y$ is a k-space, or more generally $(\beta)$ if $S \times Y$ is a $k$-space, or $(\gamma)$ if $Y$ is locally compact;

(iv) $Y$ is compact.

ProOF. For the case $S$ is a swasp parts (i) and (ii) follow from Theorem 7.5(ii) and Proposition 7.6(iii), as does part (ii) for the case $S$ is a swid.

To finish case (i) when $S$ is a swid, we note by Theorem 7.5(i) that 1 is a point of $C$-continuity for $\Pi_{K}$ for all compact $K \subset Y$, and hence a point of $C$-continuity for $\Pi$ since $Y$ is locally compact. Hence 1 is a point of continuity for $\Pi$ by Proposition 7.6(iii). Thus every $s \in S$ is a point of $C$-continuity by Theorem 7.5(iii) (with $A=B=Y$ ).

Let $Q$ be an open set with compact boundary containing $s y$, and suppose $s_{\alpha} \rightarrow s$ in $s, y_{\alpha} \rightarrow y$ in $Y$, but $s_{\alpha} y_{\alpha}$ stays outside $Q$. Since $S$ is a swid, we may assume there exist $u_{\alpha}, v_{\alpha}, w_{\alpha} \rightarrow 1$ such that $u_{\alpha} s_{\alpha}=v_{\alpha} s w_{\alpha}$ for all $\alpha$. Then $\left(u_{\alpha} s_{\alpha}\right) y_{\alpha}=$ $v_{\alpha}\left(s\left(w_{\alpha} y_{\alpha}\right)\right) \rightarrow s y$ by continuity at 1 . So for a connected neighborhood $U_{\alpha}$ of 1 we have eventually $u_{\alpha}\left(s_{\alpha} y_{\alpha}\right) \in U_{\alpha} s_{\alpha} y_{\alpha} \cap Q$ and $1\left(s_{\alpha} y_{\alpha}\right) \in U_{\alpha} s_{\alpha} y_{\alpha} \cap(Y \backslash Q)$. Thus the connected set $U_{\alpha} s_{\alpha} y_{\alpha}$ meets $\operatorname{Bd}(Q)$. Pick $t_{\alpha} \in U_{\alpha}$ such that $t_{\alpha} s_{\alpha} y_{\alpha} \in \operatorname{Bd}(Q)$. Then $t_{\alpha} s_{\alpha} y_{\alpha}$ has a convergent subnet converging to some $z \in \operatorname{Bd}(A)$. Since by definition of a swid $t_{\alpha} s_{\alpha} \rightarrow 1 s=s$ and $y_{\alpha} \rightarrow y, z=s y$ since $s$ is a point of $C$-continuity. This contradiction completes the proof of (i).

To prove part (iii), we note by Corollary 7.7 that $\Pi_{K}$ is continuous for all $K$ compact. Thus if $Q$ is any open set in $Y$, then $\Pi^{-1}(Q) \cap(S \times K)=\Pi_{K}^{-1}(Q)$ is open in $S \times K$. Thus $\Pi^{-1}(Q)$ is open. We conclude that $\Pi$ is continuous.

Part (iv) is a special case of (iii).

In [13] I posed the question of finding classes of semigroups which act continuously on compact spaces if they act separately continuously. Corollary 7.8 shows that swids on Namioka spaces are such a class.

The need to consider only locally reductive actions for swasps is something of a nuisance. Alternately one has available the following general result.

7.9 THEOREM. Suppose that $S$ is a swasp on a Namioka space, $Y$ is a uniform space, and $\Pi: S \times Y \rightarrow Y$ is an action which is locally bounded with respect to a family of compact sets $\mathcal{K}$. Then for each $t \in S$, the function $t \Pi_{K}: S \times K \rightarrow Y$ which sends $(s, y)$ to tsy is continuous for all $K \in K$.

ProOF. Let $s_{\alpha} \rightarrow s$ in $S, y_{\alpha} \rightarrow y$ in $K$, and suppose $t s_{\alpha} y_{\alpha}$ does not cluster to $t s y$. Then for some subnet $s_{\alpha} y_{\alpha} \rightarrow z \in Y$ since $\Pi$ is locally bounded. Then $t s_{\alpha} y_{\alpha} \rightarrow t z$ so $t z \neq t s y$. One now proceeds as in the proof of part (ii) of Theorem 7.5 to derive a contradiction. 
We close this section with a few pertinent comments.

7.10 REMARKS. (i) The reader is cautioned that a certain polarity exists for the results of this section. For example in Corollary 7.8 to obtain continuity of right actions, one needs a "right" swid or swasp.

(ii) The role played by Namioka property for swids is only that of an "igniter" to obtain continuity at 1 . Continuity is then tranported from 1 to points of weak subordination. If continuity at 1 is obtained by some other method, then the Namioka requirement can be eliminated.

(iii) One could slightly generalize the definition of a subordinate point by requiring that $\left\{t:(U t)^{-} \cap(U s)^{-}\right\}$be a neighborhood of $s$ for all nonempty open sets $U$. (This definition is equivalent to the other one for locally compact spaces.) The definition of strongly and weakly subordinate points could be similarly weakened. The results of this paper then go through also in this setting (the notation becomes more cumbersome and the proofs slightly more technical). A modified version of Proposition 6.4 yields that the "substantial" points in $[\mathbf{9}$ and $\mathbf{1 3}]$ are strongly subordinate points in this more general sense. Hence the results of this paper are genuine generalizations of most results there. An alternate generalization is to define a point $s$ to be subordinate if there exists a positive integer $n$ such that for any open set $U \neq \emptyset$, the set $\left\{t \in S:\right.$ there exist $t=s_{0}, s_{1}, \ldots, s_{n}=s$ such that $U_{s_{i-1}} \cap U_{s_{i}} \neq$ $\emptyset$ for $i=1, \ldots, n\}$ is a neighborhood of $s$. Again the results of this paper for subordinate points remain valid for this generalization. Whether significant examples exist satisfying this but not the other is another question.

(iv) Although products of Namioka spaces need not be Namioka spaces, in [3] a class of spaces which are not Namioka spaces, which contain locally compact and complete metric spaces, and are closed under arbitrary products are given. So swids on such spaces are productive.

8. Migrant actions. We wish to apply the results of $\S 7$ to the setting of $\S 3$ with the added hypothesis that the set of migrants is a semigroup which acts on the two spaces under consideration. To be precise, we make the following blanket assumptions throughout the remainder of this section:

(i) $S$ is a semitopological semigroup.

(ii) $X, Y$ are Hausdorff spaces, and $Z$ is a separated uniform space.

(iii) There is given a separately continuous $\Pi: X \times Y \rightarrow Z$ sending $(x, y)$ to $x * y$ such that given $x_{1} \neq x_{2}$ in $X\left(y_{1} \neq y_{2}\right.$ in $\left.Y\right)$ there exists $y \in Y(x \in X)$ such that $x_{1} * y \neq x_{2} * y\left(x * y_{1} \neq x * y_{2}\right)$.

(iv) $F: X \times S \rightarrow X$ sending $(x, s)$ to $x s$ is a right action of $S$ on $X ; G: S \times Y \rightarrow Y$ sending $(s, y)$ to $s y$ is a locally effective left action (no continuity assumptions).

(v) (Transposition law) For all $x \in X, s \in S$ and $y \in Y$,

$$
x s * y=x * s y \text {. }
$$

Before giving the first result, we have need of certain preliminaries.

8.1 Definition. Let $X_{\sigma}=(X, \sigma(X, Y))$ denote $X$ equipped with the weakest topology making $x \rightarrow x * y: X \rightarrow Z$ continuous for all $y \in Y$. Similarly let $Y_{\sigma}=$ $(Y, \sigma(Y, X))$. Note that $1_{X}: X_{\sigma} \rightarrow X$ and $1_{Y}: Y_{\sigma} \rightarrow Y$ are continuous since $\Pi$ is separately continuous. 
Let $K$ be a collection of compact subsets of $Y$ and let $X_{\mathcal{K}}$ denote $X$ equipped with the topology of uniform convergence on members $K$ (where again $X$ is thought of as a set of functions from $Y$ into $Z$ ).

8.2 LEMMA. The following statements are equivalent:

(1) $F: X_{\sigma} \times S \rightarrow X_{\sigma}$ is separately continuous.

(2) For all $x \in X$ and $y \in Y, s \rightarrow x * s y=x s * y: S \rightarrow Z$ is continuous.

(3) $G: S \times Y_{\sigma} \rightarrow Y_{\sigma}$ is separately continuous.

Furthermore $Y_{\sigma}$ is a separated uniform space.

ProOF. (1) implies (2): We have $s_{\alpha} \rightarrow s$ implies $x s_{\alpha} \rightarrow x s$ in $X_{\sigma}$; thus $x s_{\alpha} *$ $y \rightarrow x s * y$.

(2) implies (1): If $x_{\alpha} \rightarrow x$ in $X_{\sigma}$, then $x_{\alpha} * s y \rightarrow x * s y$ in $Z$ since $s y \in Y$; since $y$ was arbitrary, $x_{\alpha} s \rightarrow x s$ in $X_{\sigma}$. If $s_{\alpha} \rightarrow s$, then $x s_{\alpha} * y \rightarrow x s * y$, so $x s_{\alpha} \rightarrow x s$ in $X_{\sigma}$.

The equivalence of $(2)$ and (3) is analogous.

Let $\Omega$ be the uniformity on $Z$. The uniformity on $Y_{\sigma}$ is that inherited from the product uniformity on $Z^{X}$ (thinking of $Y$ as functions from $S$ to $Z$ ). A basis is given by

$$
F \rightarrow \mathcal{W}=\{(y, z) \in Y \times Y:(x * y, x * z) \in \mathcal{W} \text { for all } x \in F\}
$$

where $F$ is a finite subset of $X$ and $\mathcal{W} \in \Omega$.

The following result is standard.

8.3 LeMmA. The net $x_{\alpha} \rightarrow x$ in $X_{\mathcal{K}}$ if and only if for all $K \in \mathcal{K}, y_{\alpha} \rightarrow y$ in $K$ implies $x_{\alpha} * y_{\alpha} \rightarrow x * y$.

We are now ready for the main result.

8.4 THEOREM. Suppose that $S$ is a Namioka space, $\mathcal{K}$ is a collection of compact subsets of $Y$ and the action $F: X_{\sigma} \times S \rightarrow X_{\sigma}$ is separately continuous. Suppose further either:

(a) $s \in S$ is a point of subordination and the action $G: S \times Y \rightarrow S$ is locally bounded at $s$ with respect to $K$, or

(b) $S$ is a monoid with multiplication continuous at $\{1\} \times S, s \in S$ is a point of weak subordination, and $G: S \times Y \rightarrow S$ is locally bounded at 1 and $s$ with respect to K.

Then:

(i) $s$ is a point of continuity for $G_{K}: S \times K \rightarrow Y_{\sigma}$ for all $K \in \mathcal{K}$, and

(ii) the function $t \rightarrow x * t: S \rightarrow X_{\mathcal{K}}$ is continuous at $s$ for all $x \in X$.

Hence the funcitons in (i) and (ii) are continuous for the special case that $S$ is swid or swasp and the action $G$ is locally bounded at all points with respect to $K$.

Proof. (i) By Lemma 8.2, G:S $S \times Y_{\sigma} \rightarrow Y_{\sigma}$ is separately continuous. Since $1_{Y}: Y \rightarrow Y_{\sigma}$ is continuous, each $K \in \mathcal{K}$ remains compact in $Y_{\sigma}$ with the same subspace topology. Part (i) now follows from Corollary 7.7.

(ii) Let $x \in X$ and let $s_{\alpha} \rightarrow s$. If $K \in \mathcal{K}$ and $y_{\alpha} \rightarrow y$ in $K$, then $s_{\alpha} y_{\alpha} \rightarrow s y$ in $Y_{\sigma}$ by part (i). Thus $x * s_{\alpha} y_{a} \rightarrow x * s y$, i.e. $x s_{\alpha} * y_{\alpha} \rightarrow x s * y$. Part (ii) follows from Lemma 8.3.

We wish to transport weak continuity at 1 to other points. For this purpose we need the stronger notion of a strongly subordinate point (Definition 6.1). 
8.5 TheOREM. Assume either that (i) for all $t \in S$, the left action $G: S \times Y_{\sigma} \rightarrow$ $Y_{\sigma}$ is locally bounded at $t$ with respect to $\mathcal{F}$, the collection of singleton subsets, or that (ii) for all $y \in Y, G_{y}: S \rightarrow Y_{\sigma}$ sending $t$ to ty is continuous. If for fixed $x \in X$, the function $F_{x}: S \rightarrow X_{\sigma}$ sending $t$ to $x t$ is continuous at 1 and if $s$ is a strongly subordinate point, then $F_{x}$ is continuous at $s$. Hence if every point is strongly subordinate (e.g. $S$ is a stip), then $F_{x}$ is weakly continuous.

Proof. (i) Let $s_{\alpha} \rightarrow s$, let $y \in Y$, and let $\mathcal{W} \in \Omega$, the uniformity on $Z$. By local boundedness of $G: S \times\{y\} \rightarrow Y_{\sigma}$ at $s$, we have $s_{\alpha} y \rightarrow z$ in $Y_{\sigma}$ for some subnet.

Pick a symmetric $U \in \Omega$ such that $\mathcal{U}^{4} \subset \mathcal{W}$. Pick an open set $N$ containing 1 such that $x N * s y \subset \mathcal{U}[x * s y]$ and $x N * z \subset \mathcal{U}[x * z]$ (by continuity at 1). Then there exists $u \in N$ and $\left\{v_{\alpha}\right\} \subset N$ such that eventually $u s_{\alpha}=v_{\alpha} s$.

Then eventually $\left(x s_{\alpha} * y=x * s_{\alpha} y, x * z\right) \in \mathcal{U}$ (since $s_{\alpha} y \rightarrow z$ in $\left.Y_{\alpha}\right) ;(x *$ $z, x u * z) \in \mathcal{U}$ (since $u \in N$ ); eventually $\left(x u * z, x u * s_{\alpha} y\right) \in \mathcal{U}$ ( since $s_{\alpha} y \rightarrow z$ ); $\left(x u * s_{\alpha} y=x v_{\alpha} * s y, x * s y\right) \in \mathcal{U}$ (since $u s_{\alpha}=v_{\alpha} s$ and $\left.v_{\alpha} \in N\right)$. Thus eventually $\left(x s_{\alpha} * y, x s * y\right) \in \mathcal{U}^{4} \subset \mathcal{W}$. Since $y$ and $\mathcal{W}$ were arbitrary, $x s_{\alpha} \rightarrow x s$ in $X_{\sigma}$.

(ii) $x s_{\alpha} * y=x * s_{\alpha} y \rightarrow x * s y=x s * y$.

9. Actions on locally convex spaces. The action of topological groups on locally convex spaces, closely related with representation theory, has an extensive literature. A good summary of the state of the art up to that time may be found in [15]. The two techniques found there involve "smoothing" via integration and Baire category techniques. The latter extend to the semigroup setting, and have been skillfully exploited by Helmer $[8,9]$. The special class of semigroups called "stips" and their actions on certain convex spaces have been considered by Sleijpen $[\mathbf{2 3}]$ and Dzinotyiweyi [5]. It is our aim in this section to wed these two approaches.

In this section let $X$ denote a locally convex separated topological vector space (over the reals or complexes). We denote the dual space of continuous linear functionals by $Y$. There is induced a bilinear form $(x, f) \rightarrow\langle x, f\rangle=f(x)$ from $X \times Y$ into the base field. We assume always that $Y$ is equipped with the weak topology $\sigma(Y, X)$, and we let $X_{\sigma}$ stand for $X$ equipped with the weak topology $\sigma(X, Y)$.

Now from the basic theory of locally convex spaces, if $B$ denotes the family of equicontinuous subsets of $Y$, then for any $B \in B$ the bipolar $B^{\circ \circ}$ (which is the closed convex hull of $B \cup\{0\})$ is compact in $Y\left(=Y_{\sigma}\right)$ and is again in $B$; furthermore; $X=X_{\mathrm{B}}$, where $X_{\mathrm{B}}$ stands for $X$ equipped with the topology of uniform convergence on members of $B$. (The reader is referred to $\S \S I I I .4$ and IV.1, 2 of $[\mathbf{2 2}]$ for the basic material on locally convex spaces used in this section.)

Let $S$ be a semitopological semigroup which acts on $X$ on the right, $(x, s) \rightarrow$ $x s: X \times S \rightarrow X$, such that each $s \in S$ acts as a continuous linear transformation on $X_{\sigma}$. Then there is defined a dual left action of $S$ on $Y$ which satisfies $\langle x s, y\rangle=$ $\langle x, s y\rangle$ for all $x \in X, s \in S, y \in Y$. (Note that this is just the transposition law with $\langle$,$\rangle playing the role of *$.) Furthermore each $s \in S$ acts as a continuous linear transformation on $Y$ (see Lemma 8.2).

The following is a variant of Theorem 3.2 of [9]. 
9.1 ThEOREM. Suppose $\Pi: X \times S \rightarrow X$ is a linear action of the semigroup $S$ on $X$ which is separately continuous for $X=X_{\sigma}$, where $S$ is a Namioka space. Suppose further either that:

(a) $p$ is a subodinate point of $S, \Pi$ is continuous at $(0, p)$, and the induced action $G: S \times Y \rightarrow Y$ is locally effective at $p$, or

(b) $S$ is a monoid with multiplication continuous at $\{1\} \times S, p$ is a weakly subordinate point, and $\Pi$ is continuous at $(0,1)$ and $(0, s)$.

Then

(i) $(s, y) \rightarrow$ sy: $S \times Y \rightarrow Y$ is locally bounded at 1 and $p$ with respect to the compact members of $B$;

(ii) the mapping $s \rightarrow x s: S \rightarrow X_{B}$ is continuous at $p$ for each $x \in X$;

(iii) $p$ is a point of continuity for the mapping $\Pi: X_{B} \times S \rightarrow X_{B}$.

ProOF. We present case (b); case (a) is similar. Let $K$ be the closed convex circled equicontinuous subsets of the dual $Y$. Then each $B \in \mathcal{K}$ is compact in $Y$ and $B=B^{\circ \circ}$. Let $B \in \mathcal{K}$. Then $B^{\circ}$, the polar of $B$, is a neighborhood of 0 in $X_{B}$. By hypothesis there exist neighborhoods $U$ of 0 and $V$ of $p$ such that $U V \subset B^{\circ}$. Since $X_{B}$ is locally convex, $U$ can be chosen equal to its closed convex circled hull; thus $U^{\circ \circ}=U$. Then $U^{\circ}$ is equicontinuous and closed, hence in $\mathcal{K}$. We have

$$
V B=V B^{\circ \circ} \subset V(U V)^{\circ} \subset U^{\circ}
$$

the last inclusion follows since

$$
|\langle u, v y\rangle|=|\langle u v, y\rangle| \leq 1
$$

for all $u \in U, v \in V$, and $y \in(U V)^{\circ}$. The same argument works for $p=1$.

(ii) By Theorem 8.4,s $\rightarrow x s: S \rightarrow X_{K}$ is continuous at $p$ for all $x \in X$. Since $K \subset B$ and each member of $B$ is contained in a member of $K, X_{K}=X_{B}$.

(iii) Let $x_{\alpha} \rightarrow x$ in $X_{B}$ and $s_{\alpha} \rightarrow p$ in $S$. Then $x_{\alpha}-x \rightarrow 0$, so $\left(x_{\alpha}-x\right) s_{\alpha}=$ $x_{\alpha} s_{\alpha}-x s_{\alpha} \rightarrow 0$ by continuity at $(0, p)$. By part (ii) $x s_{\alpha} \rightarrow x p$ in $X_{B}$. The last statements imply that $x_{\alpha} s_{\alpha} \rightarrow x p$.

9.2 COROLlaRY. Let $\Pi: E \times S \rightarrow E$ be a linear action of a swid $S$ on a locally convex space $E$ such that $\Pi$ is continuous at $(0, s)$ for all $s \in S$, and assume $S$ is a Namioka space.

(i) If $x \in E$ is a weakly continuous vector (i.e. $s \rightarrow x s: S \rightarrow E_{\sigma}$ is continuous), then $x$ is a continuous vector $(s \rightarrow x s: S \rightarrow E$ is continuous).

(ii) The set of (weakly) continuous vectors forms a closed subspace $X$ of $E$ and the restriction of the action $\Pi \mid: X \times S \rightarrow X$ is continuous.

Proof. Let $X=\{u \in E: u$ is weakly continuous $\}$. A routine verification yields that $X$ is a linear subspace containing $x$ and that $X$ is invariant under the action of $S$.

We show $X$ is closed in $E$. Suppose $v$ is in the closure of $X$ and $s_{\alpha} \rightarrow s$ in $S$. Then

$$
v s-v s_{\alpha}=(v-y) s+\left(y s-y s_{\alpha}\right)+(y-v) s_{\alpha} \quad \text { where } y \in X .
$$

By continuity at $(0, s),(v-y) s$ and $(y-v) s_{\alpha}$ approach 0 in $E$ as $y \in X$ is chosen near $v$ and $s_{\alpha}$ near $s$. Since $y s_{\alpha} \rightarrow y s$ weakly, we conclude $v s-v s_{\alpha}$ can be made weakly near 0 . Hence $v$ is weakly continuous. 
The action of $S$ restricted to $X$ remains continuous at all points $(0, s), s \in$ $S$, and each $s$ acts continuously since it does at 0 . Finally every vector in $X$ is weakly continuous with respect to the dual $Y$ of $X$ since every continuous linear functional on $X$ extends to one on $E$ by the Hahn-Banach theorem. Hence the action $X_{\sigma} \times S \rightarrow X_{\sigma}$ is separately continuous (continuous linear operators on $X$ are continuous on $X_{\sigma}$ ).

By part (ii) of Theorem 9.1 applied to $X$, we conclude that $s \rightarrow x s: S \rightarrow X$ is continuous. Since $X$ has the subspace topology, $x$ is a continuous vector in $E$.

The remainder of the corollary now follows from part (iii) of Theorem 9.1.

We now recover (a slight generalization of) the main result of Dzinotyiweyi in [5] (see also, [23, Theorem 3.13 and 25]).

9.3 COROLlaRY. Let $S$ be a swid on a Namioka space which acts on the right as linear transformations on a normed space $E$. If $\|x s\| \leq\|x\|$ for all $x \in E, s \in S$, then a weakly continuous vector is a continuous vector.

Proof. The condition $\|x s\| \leq\|x\|$ for all $x \in E$ implies that $\Pi: E \times S \rightarrow E$ is continuous at $(0, s)$ and that $x \rightarrow x s: E \rightarrow E$ is continuous. This corollary now follows from the preceding one.

The next result closely parallels one of Helmer's [9, Theorem 4.2].

9.4 COROLlARY. Let $\Pi: E_{\sigma} \times S \rightarrow E_{\sigma}$ be a separately continuous linear action of a locally compact swid $S$ on a barrelled space $E$. Then $\Pi: E \times S \rightarrow E$ is continuous.

Proof. Each $s \in S$ acts as a weakly continuous, hence continuous, operator on $E$. For $s \in S$, let $N$ be a compact neighborhood of $s$. Then for each $x \in E$, $x N$ is weakly compact, hence weakly bounded, and thus bounded. Hence by the Uniform Boundedness Principle [22, p. 83] the collection of linear transformations corresponding to $N$ is equicontinuous. It follows that $(x, t) \rightarrow x t: E \times N \rightarrow E$ is continuous at $(0, s)$. The desired conclusion now follows from Theorem 9.1.

9.5 REMARK. Analogues for Corollaries 9.2, 9.3, and 9.4 exist for swasps provided the induced actions on the appropriate dual spaces are locally effective. In 9.2 and 9.3 the appropriate dual space is the dual of the space of weakly continuous vectors. In 9.4 it is simply the dual of $E$ (see Theorem 3.2 of [9] for appropriate conditions for this latter case).

We give some examples of results which remain valid for swasps.

9.6 COROLlARY. Let $\Pi: E \times S \rightarrow E$ be a linear action of a swasp $S$ on a locally convex space $E$ such that $\Pi$ is continuous at $(0, s)$ for all $s \in S$. Assume $S$ is a Namioka space and that $x \in E$ is a weakly continuous vector.

(i) For each $t \in S$, xt is a continuous vector.

(ii) If the vector $x$ is $S$-generated, i.e. if $x$ is the closure of the vector subspace generated by $x S$, then $x$ is a continuous vector.

(iii) If $S=\bigcup_{i=1}^{n} e_{i} S$ where each $e_{i}$ is an idempotent, then $x$ is a continuous vector.

Proof. (i) Let $X=\{u \in E: u$ is weakly continuous $\}$. As in Corollary $9.2, X$ is a closed linear subspace of $E$ containing $x$ which is invariant under the action of $S$, and the induced action on its dual $Y$ is separately continuous and locally bounded 
with respect to $K$, the compact equicontinuous subsets of $Y$. By Theorem 7.9 for $t \in S,(s, y) \rightarrow t s y: S \times K \rightarrow Y$ is continuous for all $K \in \mathcal{K}$. Thus if $s_{\alpha} \rightarrow s, x t s_{\alpha}$ converges uniformly to $x$ ts on $K$. Since $X$ has the topology of uniform convergence on members of $\mathcal{K}, x t$ is a continuous vector in $X$ and hence in $E$.

(ii) A modified version of the first part of the proof of Corollary 9.2 yields that the set of continuous vectors is a closed vector subspace of $E$. The result then follows from part (i).

(iii) By Proposition 7.2 the action on the dual of the weakly continuous vectors is locally effective. Hence the proof of Corollary 9.2 goes through.

Corollary 9.6 is a generalization of some of the results of Sleijpen in [25]. Part (iii) gives an affirmative answer to a question he raises there. He gives a counterexample for the case finitely many idempotents are replaced by countably many.

For strongly subordinate points one obtains analogues of Sleijpen's results [23].

9.7 THEOREM. Let $\pi: E \times S \rightarrow E$ be a linear action of a monoid $S$ on a locally convex space $E$ such that each $t \in S$ acts as a continuous linear transformation.

(i) If $s \in S$ is a strongly subordinate point, if the action is continuous at $(0, s)$, and if for $p \in E, t \rightarrow p t: S \rightarrow E_{\sigma}$ is continuous at 1 , then it is continuous at $s$.

(ii) If every point of $S$ is strongly subordinate, $S$ is a Namioka space, and the action is continuous at $\{0\} \times S$, then if the vector $p$ is weakly continuous at 1 , it is a continuous vector.

ProOF. (i) Suppose $s \in S$ is strongly subordinate and $\Pi$ is continuous at $(0, s)$. By part (i) of Theorem 9.1 the dual action $S \times E^{\prime} \rightarrow E^{\prime}$ is locally bounded at $(s, y)$ for all $y \in E^{\prime}$. (That is, $U y$ has compact closure for some neighborhood $U$ of $s$. The additional hypotheses of that theorem were not used in part (i).) By Theorem 8.6, $t \rightarrow p t: S \rightarrow E_{\sigma}$ is continuous at $s$ (only the local boundedness at $s$ was used in the proof).

(ii) This part follows from part (i) and Corollary 9.2.

Corollary 9.2 and Theorem 9.7 provides nice (one-sided) generalizations of the main results of Sleijpen in $[\mathbf{2 2}]$ of the action on the measure algebra.

10. Further questions. We close the paper with what appear to be some interesting problems for further research.

1. Characterize those semitopological semigroups for which every separately continuous action is locally effective (or at least identify classes of such).

2. Weak subordination is a sufficient condition on a point for it to be a point of continuity for separately continuous actions on compact spaces. Find interesting necessary conditions.

3. Let $S$ be a compact semitopological semigroup with a dense subgroup. Suppose $g_{\alpha} \rightarrow x$, a regular element. Does $g_{\alpha}^{-1}$ converge to an inverse for $x$ ?

4. A locally compact semigroup $S$ acts on its measure algebra both on the left and right. Sleijpen [22] showed that if $S$ is a stip, then the weakly continuous vectors for each action coincide. Is this (or some generalization thereof) provable by the techniques of this paper?

5. If $S \times Y \rightarrow Y$ is a(n) (effective) separately continuous actions of a product $S$ of finite semigroups on a compact space $Y$, is the action continuous (for finite monoids this is true by $\S 7)$ ? 


\section{REFERENCES}

1. J. F. Berglund, Weakly almost periodic flows (to appear).

2. J. F. Berglund and K. H. Hofmann, Compact semitopological semigroups and weakly periodic functions, Lecture Notes in Math., vol. 42, Springer-Verlag, Heidelberg, 1967.

3. J. P. R. Christensen, Joint continuity of separately continuous functions, Proc. Amer. Math. Soc. 82 (1981), 455-461.

4. A. H. Clifford and G. B. Preston, The algebraic theory of semigroups. I, Math. Surveys, vol. 7, Amer. Math. Soc., Providence, R.I., 1961.

5. H. A. M. Dzinotyiweyi, Weak and norm continuity of semigroup actions on normal linear spaces, Quart. J. Math. Oxford Ser. (2) 33 (1982), 85-90.

6. R. Ellis, Locally compact transformation groups, Duke Math. J. 24 (1957), 119-126.

7. G. Hansel and J. P. Troallic, Points de continuite a gauche d'une action de semigroupe, Semigroup Forum 26 (1983), 205-214.

8. D. Helmer, Joint continuity of affine semigroup actions, Semigroup Forum 21 (1980), 153-165.

9. __ Continuity of semigroup actions, Semigroup Forum 23 (1981), 153-188.

10. K. H. Hofmann and P. S. Mostert, Elements of compact semigroups, Merrill, Columbus, Ohio, 1966.

11. P. Kenderov, Dense strong continuity of pointwise continuous mappings, Pacific J. Math. 89 (1980), 111-130.

12. J. D. Lawson, Joint continuity in semitopological semigroups, Illinois J. Math. 18 (1974), 175-185.

13. __ Additional notes on continuity in semitopological semigroups, Semigroup Forum 12 (1976), 165-280.

14. J. D. Lawson and B. Madison, On congruences and cones, Math. Z. 120 (1971), 18-24.

15. R. T. Moore, Measurable, continuous and smooth vectors for semigroups and group representations, Mem. Amer. Math. Soc. No. 78 (1968).

16. I. Namioka, Separate continuity and joint continuity, Pacific J. Math. 51 (1974), 515-531.

17. W. Ruppert, Notes on compact semigroups with identity, Semigroup Forum 14 (1977), 199-234.

18. __ On semitopological compactification of non-abelian groups, Illnois J. Math. 26 (1982), 388411.

19. __ A structural theory of compact semitopological semigroups (to appear).

20. C. Ryll-Nardzweski, On fixed points of semigroups of endomorphisms of linear spaces, (Proc. 5th Berkley Sympos. Math. Stat. and Prob., 1965), vol. II, Part 1, Univ. of California Press, Berkeley, 1966.

21. J. Saint Raymond, Jeux topologiques et espaces de Namioka, Proc. Amer. Math. Soc. 87 (1983), 499-504.

22. H. H. Schaefer, Topological vector spaces, Springer-Verlag, Heidelberg, 1971.

23. G. L. G. Sleijpen, Locally compact semigroups and continuous translations of measures, Proc. London Math. Soc. (3) 37 (1978), 75-97.

24. __ Emaciated sets and measures with continuous translations, Proc. London Math. Soc. (3) 37 (1978), 98-119.

25. _ The action of a semigroup on a space of bounded Radon measures, Semigroup Forum 23 (1981), 137-157.

26. J.-P. Troallic, Espaces fonctionnels et théorèmes de I. Namioka, Bull. Soc. Math. France 107 (1979), 127-137.

27. __ Semigroupes semitopologiques et presqueperiodicite, (Proc. Conf. Semigroups, Oberwolfach 1981), Lecture Notes in Math., vol. 998, Springer-Verlag, Heidelberg, 1983, pp. 239-251.

Department of Mathematics, Louisiana State University, Baton Rouge, LOUISIANA 70803-4981 